Results Participants were 10\% affluent, $65 \%$ from middle-class social groups and $25 \%$ of relatively deprived minority status. Two - thirds of parents regard themselves as overprotective and highly anxious, $32 \%$ present responsible and caring and $1 \%$ tend to be indifferent. $19 \%$ of parents initially refused admission. The major pre-admission emotions are self- blame, anger and fear, positively associated to previous hospital stays. Stress and discomfort dominate through the in-hospital phase, during which 23\% of parents experienced psychosomatic symptoms. Negative emotions become reversed when the need for information by doctors and nurses is satisfied. Fathers expressed less effectively both positive and negative emotions.

Limitations Parents of children with chronic diseases were excluded from the study.

Conclusions Children's sickness and hospitalisation disorganizes the family, resulting in parental emotional disturbance, confusion and uncertainty. Stress-related emotions are better coped with, when parents feel they are respectfully treated and adequately fed-back by the hospital personnel.

\section{PO-0381 KARTAGENER SYNDROME ASSOCIATED TO NEPHROPATHY: A BROADER SPECTRUM OF CILIOPATHY?}

${ }^{1}$ E Veiga, ${ }^{1} \mathrm{~A}$ Veiga, ${ }^{1} \mathrm{~J}$ Llerena Junior, ${ }^{1} \mathrm{C}$ Serao, ${ }^{1} \mathrm{C}$ Mochdece, ${ }^{2} \mathrm{P}$ Oliveira Goncalves, ${ }^{2} \mathrm{~S}$ Teixeira, ${ }^{2}$ R Semenzim. ${ }^{1}$ Pediatria, Faculdade Medicina de Petrópolis, Petrópolis, Brazil; ${ }^{2}$ Graduação Em Medicina, Faculdade Medicina de Petrópolis, Petrópolis, Brazil

\subsection{6/archdischild-2014-307384.1027}

Introduction Kartagener Syndrome (KS), an autosomal recessive disorder, subgroup of primary ciliary dyskinesia, is characterised by situs inversus, bronchiectasis and chronic sinusitis.

Objective To report a case of KS and associated nephropathy, diagnosed at 6 years in Hospital Alcides Carneiro (HEAC).

Method Descriptive and observational evolution of MESF, hospitalised in the paediatric ward of the HEAC, with abdominal pain and nephritic proteinuria. Clinical diagnosis of SK was established only at 6 years, despite dextrocardia diagnosed at 3 months and several subsequent hospitalizations due to respiratory infections. Nephritis was identified at 4 years, slowly progressive and without specific etiological cause determinated. Tomography showed bronchiectasis and situs inversus totallis. No identifiable chromosomal abnormalities seen in conventional karyotyping and no molecular study could be performed. Clinical diagnosis, however, made possible hearing loss and lung infections prophylaxis and genetic counselling. Although there are some reports of genitourinary abnormalities associated with SK, it's important to determine if $\mathrm{KS}$ is the specific cause of nephropathy, due the intimate relationship of other ciliopathys with renal disease, as observed in Bardet-Biedl syndrome and Meckel syndrome. In this patient the aetiology of nephropathy is unclear so far, and most common causes have been discarded.

Conclusion Factors such as low incidence estimated at 1:17000 births and pathophysiological complexity are considered contributory to late diagnosis, worsening of pulmonary disease and consequently worse prognosis. We couldn't perform molecular diagnosis in the several genes related to SK, which could provide preimplantation diagnosis and establish the best genotype-phenotype, especially in correlation with nephropathy.

\section{PO-0382 DEVELOPMENTAL DELAY IN TRISOMY 21 CHILDREN FROM ROMANIA}

${ }^{1}$ RM Vlad, ${ }^{2} \mathrm{P}$ Grigorescu-Sido, ${ }^{2} \mathrm{~S}$ Bucerzan, ${ }^{2} \mathrm{C}$ Al-Khzouz, ${ }^{2} \mathrm{Nascu},{ }^{1} \mathrm{D}$ Oraseanu. 'Pediatrics, "Grigore Alexandrescu" Emergency Children's Hospital "Carol Davila" University of Medicine and Pharmacy, Bucharest, Romania; ${ }^{2}$ Medical Genetics, "Iuliu Hatieganu" University of Medicine and Pharmacy, Cluj Napoca, Romania

\subsection{6/archdischild-2014-307384.1028}

Aims Trisomy 21 is the most frequent autosomal chromosomopathy. The developmental delay is a constant finding. This study aimed to evaluate the auxological parameters of Romanian trisomy 21 patients and to correlate them with the cytogenetic findings.

Material and method We conducted an observational study on 136 patients with cytogenetically confirmed trisomy 21 that were evaluated recording their auxological parameters. The nutritional status of the patients was evaluated using growth charts from WHO until 5 years and from CDC over 5 years. For infants, the ponderal index was used. For patients $\geq 1$ year, we used growth charts for weight and BMI and standard deviation score for height. The growth parameters were statistically evaluated considering three age groups (newborn, infant, child $\geq 1$ year) and the cytogenetic anomalies.

Results $91.2 \%$ of patients had regular trisomy 21 . The mean birth weight was 2904grams and the mean birth length $49,6 \mathrm{~cm}$. $80 \%$ of the infants associated protein-caloric malnutrition; the length was affected in $54.9 \%$ of 1 month - 1 year patients. $49.4 \%$ of patients $\geq 1$ year were underweight, $37.03 \%$ were overweight and $75.3 \%$ associated short-stature. Only $14.5 \%$ of patients $<1$ year and $8.6 \%$ of patients $\geq 1$ year had a normal nutritional status. No significant differences were found in relation to the cytogenetic diagnosis.

Conclusions The somatic delay varied in relation to age. In infants underweight dominated. In children $\geq 1$ year short-stature was the main finding and one third of patients were overweight. Only a reduced number of trisomy 21 children had normal auxological parameters.

\section{PO-0383 MONITORING IGG ON ORAL FLUID FOR THE MANAGEMENT OF CHILDREN AT RISK FOR CONGENITAL TOXOPLASMOSIS}

${ }^{1} \mathrm{M}$ Wallon, ${ }^{2} \mathrm{E}$ Chapey, ${ }^{3} \mathrm{~V}$ Meroni, ${ }^{4} \mathrm{~F}$ Kieffer, ${ }^{5} \mathrm{P}$ Garcia-Meric, ${ }^{6} \mathrm{C}$ L'Ollivier, ${ }^{1} \mathrm{~F}$ Peyron. 1 Institut de Parasitologie Et de Mycologie Médicale, Hôpital de La Croix-Rousse, Lyon, France; ${ }^{2}$ Faculté de Médecine Et de Maïeutique Lyon Sud, UCBL, Lyon, France; ${ }^{3}$ Fondazione IRCCS, Policlino San Matteo, Pavia, Italy; ${ }^{4}$ Service de Néonatalogie, Hôpital Trousseau, Paris, France; ${ }^{5}$ Service de Médecine Néonatale, Hôpital de La Conception, Marseille, France; ${ }^{6}$ Laboratoire de Parasitologie Mycologie Médicale, Hôpital de La Timone, Marseille, France

\subsection{6/archdischild-2014-307384.1029}

Background and aims Despite progress made for the diagnosis of congenital Toxoplasma infection in utero and at birth, serological follow-up in first year of life remains required to exclude or confirm congenital infection. To reduce the constraints of this follow-up, we developed a test to detect anti-Toxoplasma IgG in oral fluid.

Methods In 362 patients referred for Toxoplasma serology oral fluid was collected on two micro-sponges in parallel to blood sampling. A pilot study on 212 patients aged $>15$ months (274 samples) was performed to validate sampling procedures and develop an in-house indirect ELISA for the detection of 